\title{
Outsourcing: beneficios vs. riesgos
}

\author{
DIANA CRISTINA BEDOYA-GÓMEZ ${ }^{a}$
}

pp. 101-112

RESUMEN El Outsourcing, también llamado tercerización, subcontratación o externalización, es el proceso de contratación de un tercero para que realice una actividad del negocio, durante la lectura de esta nota técnica se podrá encontrar los beneficios y riesgos de esta práctica que cada vez más se vuelve recurrente en las organizaciones. Para la investigación se realizó una búsqueda en fuentes primeras y secundarias, donde fue fundamental la información proporcionada por empresarios que sirven de ejemplos para ilustrar de manera clara cada elemento.

Entre los beneficios encontrados del outsourcing se describen: contratar con proveedores que ofrecen menores costos, reducir o eliminar costos fijos, concentrarse en las actividades clave, conocimiento, experiencia y tecnología del proveedor; en el caso de los riesgos se describen ampliamente los siguientes: convertir a un proveedor en competidor, pérdida de conocimiento y habilidades, dependencia del proveedor, incremento de los costos, afectación del clima laboral y incumplimiento con las expectativas de calidad y de tiempos de entrega.

El outsourcing es una estrategia que se debe analizar con detenimiento, puesto que, si bien son muchos los beneficios, también genera riesgos que deben ser identificados, medidos, valorados y tratados. La decisión no debe justificarse en elementos únicamente financieros, se debe incluir en el análisis, la importancia del proceso para la empresa, la experiencia del proveedor, los acuerdos de negociación y todos aquellos elementos cualitativos que permitan tomar la decisión alineada con la estrategia de la organización.

\section{HISTORIA DEL ARTÍCULO}

¿CÓMO CITAR?:

Bedoya-Gómez, D.C. (2018).

Outsourcing: beneficios vs. Riesgos. Perspectiva Empresarial, 5(2), 101-112. http://dx.doi. org/10.16967/rpe.v5n2a7

RECIBIDO: 4 de agosto de 2017 APROBADO: 18 de junio de 2018

CORRESPONDENCIA:

Diana Cristina Bedoya-Gómez, Cl. 77 Sur No 40-165, Sabaneta, Antioquia. Colombia.

PALABRAS CLAVE externalización, outsourcing, subcontratación, riesgos, tercerización.

a Magíster en Administración Financiera, docente de tiempo completo en Fundación Universitaria CEIPA, Colombia. Correo electrónico: diana.bedoya@ceipa.edu.co 


\section{¿CÓMO CITO EL ARTÍ́CULO? HOW TO CITE THIS PAPER?}

CHICAGO:

Bedoya-Gómez, Diana Cristina. 2018. "Outsourcing: beneficios vs. Riesgos". Perspectiva Empresarial 5(2): 101-112. http://dx.doi.org/10.16967/ rpe.v5n2a7

MLA: Bedoya-Gómez, Diana Cristina. "Outsourcing: beneficios vs. Riesgos". Perspectiva Empresarial 5.2 (2018): 101-112. Digital. http://dx.doi. org/10.16967/rpe.v5n2a7

\section{Outsourcing: Benefits vs. Risks}

ABSTRACT Outsourcing, also called subcontracting, is the process of engaging a third party to carry out a business activity. In reading this technical note, you may find the benefits and risks of this practice that has become increasingly recurring in organizations. For the research, a search was made in initial and secondary sources, where the information provided by businessmen who serve as examples to clearly illustrate each item was essential.

Some of the benefits of outsourcing are as follows: hiring the suppliers that offer lower costs, reducing or eliminating fixed costs, and focusing on the supplier's key activities, knowledge, experience and technology. In the case of risks, the following are broadly described: turning a supplier into a competitor, loss of knowledge and skill, dependence on the supplier, increased costs, impact on the work environment, and failure to meet quality expectations and delivery times.

Outsourcing is a strategy that must be thoroughly analyzed because, while it entails many benefits, there are also risks that must be identified, measured, assessed and managed. The decision should not be justified by economic aspects only; the analysis should include the importance of the process for the company, the supplier's experience, the business agreements and any other aspect that allows to make a decision in accordance with the organization's strategy.

KEYWORDS benefits, outsourcing, risks, subcontracting.

\section{Terceirização: benefícios vs. riscos}

RESUMO A terceirização, também chamada de outsourcing, subcontratação ou externalização, é o processo de contratação de um terceiro para realizar uma atividade do negócio. Durante a leitura desta nota técnica será possível encontrar os benefícios e riscos dessa prática que se torna cada vez mais frequente nas organizações. Para a pesquisa foi realizada uma análise em fontes primárias e secundárias, na qual foi fundamental a informação proporcionada por empresários, que serve como exemplo para ilustrar, de maneira clara, cada elemento.

Entre os benefícios da terceirização que foram descritos estão: contratar com fornecedores que oferecem menores custos, reduzir ou eliminar custos fixos, concentrar-se nas atividades fundamentais, conhecimento, experiência e tecnologia do fornecedor. No caso dos riscos, são descritos amplamente os seguintes: converter o fornecedor em concorrente, perda de conhecimento e habilidades, dependência do fornecedor, aumento dos custos, impacto no clima laboral e contrariar as expectativas de qualidade e de tempo de entrega.

A terceirização é uma estratégia que deve ser analisada com atenção, posto que, ainda que sejam muitos os benefícios, também gera riscos que devem ser identificados, medidos, avaliados e tratados. A decisão não deve ser justificada em elementos unicamente financeiros. É necessário incluir na análise a importância do processo para a empresa, a experiência do fornecedor, os acordos de negociação e todos os demais elementos qualitativos que possam permitir que uma decisão alinhada à estratégia da organização seja tomada.

PALAVRAS CHAVE Benefícios, externalização, outsourcing, riscos, subcontratação, terceirização. 


\section{Introducción}

A menudo, las empresas se encuentran frente a la disyuntiva de fabricar ellos mismos un componente o contratar a un tercero para que lo produzca. Asimismo, una empresa puede decidir contratar un servicio, como, por ejemplo, el transporte, la contabilidad o la atención al cliente, o llevar a cabo esta actividad por sí misma. El outsourcing aplica tanto a la decisión de pedir a alguien que fabrique un componente como que preste un servicio.

El outsourcing — también denominado "tercerización", "subcontratación" o "externalización" es el proceso de contratación de un tercero para que realice una actividad del negocio que, por diferentes razones, no se lleva a cabo de manera interna. En otros términos, es delegar la ejecución de una de las actividades, de los procesos o los productos desarrollados inicialmente por la empresa, a un tercero o proveedor. Esta es una tendencia muy recurrente en empresas grandes y pequeñas de todo el mundo, e implica cambios en los procesos, la infraestructura, la tecnología, los recursos financieros e, incluso, en la cultura.

Nike Inc., la multinacional estadounidense, es un ejemplo claro de outsourcing. Esta reconocida empresa de calzado, ropa, equipo y otros artículos deportivos centraliza solo las actividades de diseño e investigación; todas las demás funciones que necesita para producir y comercializar sus productos las subcontrata con empresas de todo el mundo. Entre las ventajas de esta práctica para Nike se destaca la posibilidad de concentrarse en su razón de ser. Asimismo, reducir los costos de producción -dado que, en general, busca los servicios de outsourcing en países que impliquen una producción de bajo costo-, producir de una manera más eficiente y trabajar de forma flexible, dada la estructura más pequeña y menos burocrática que ha logrado establecer (Cepeda, 2012).

El outsourcing no solo se aplica a la producción, también es posible emplearlo en áreas como mercadeo, finanzas, gestión humana, logística y tecnología de la información (TI). En el área financiera, por ejemplo, para servicios relacionados con la planeación y la declaración tributaria se puede desarrollar outsourcing. Lo mismo sucede con el área de gestión humana, ya que procesos como el reclutamiento y la selección de personal se pueden contratar con empresas especializadas en estas tareas.
El outsourcing es una práctica popular desde hace varios años y cada vez abarca más sectores económicos, tales como los relacionados con tecnología informática, manufactura de aparatos electrónicos, industria automotriz o sector financiero, entre otros. Durante los años 2012, 2013 y 2014, Colombia se destacó como uno de los 30 destinos más importantes de outsourcing/ off-shore del mundo, según la consultora Gartner. "Cada vez más, diversos sectores de la economía están aprendiendo a comprar servicios de outsourcing de una mejor manera y apalancados con metodologías de talla mundial", indica Juan Pablo Llano, director de Mercadeo y Alianzas de Carvajal Tecnología y Servicios (Revista Dinero, 2014).

Un caso de éxito para Colombia es la industria del business process outsourcing (ВРо, "procesos de tercerización"), la cual mueve 6,16 billones de pesos al año en Colombia y crece al 10,27\%. En Medellín, durante el 2016 se dieron dos grandes movimientos para consolidar el negocio del BPO: la fusión de Compuredes y Enlace Operativo, lo que dio origen a Arus, con inversiones en 1200 millones de pesos en cambio de marca y 1,7 millones de dólares para renovación tecnológica; asimismo, la adquisición de Allus Latinoamérica por parte del grupo español Konecta, por 170 millones de euros (Suárez, 2016).

Esta industria presenta un gran crecimiento en ingresos, tamaño, calidad y sofisticación de los servicios. Los sectores con mayor potencial de crecimiento son call centers y soporte técnico, el desarrollo de software y los centros de servicio compartidos (outsourcing de back office), BPO (procesos de tercerización), ITO y KPO, así como servicios de nube privada (Suárez, 2016).

La presente nota técnica tiene como objetivo explicar, en un lenguaje sencillo, los beneficios y los riesgos del outsourcing, así como utilizar ejemplos de empresas colombianas para su ilustración.

\section{Beneficios del outsourcing}

El outsourcing tiene diferentes puntos favorables que permiten mejorar la administración de los costos, focalizarse en las actividades clave del negocio, optimizar los procesos, ampliar el conocimiento, especializarse, emplear mayor tecnología o incrementar la eficiencia. A continuación, se describirán en detalle cada uno los beneficios, 
con especial énfasis en ejemplos de empresas de Colombia.

\section{Contratar con proveedores que ofrecen menores costos}

El primer beneficio del outsourcing es la posibilidad de contratar con proveedores que pueden ofrecer un producto o servicio a un precio menor al que le costaría a la empresa realizarlo internamente. El proveedor es capaz de lograr costos más bajos gracias a la economía de escala; es decir, el manejo de altos volúmenes de producción, el acceso a insumos más baratos y la adquisición de tecnologías, entre otros.

Una empresa que presta servicios de outsourcing y contrata con varios clientes el desarrollo de un componente similar, logra altos volúmenes de producción beneficiándose de la estructura de costos. Se pueden lograr descuentos por volumen, alcanzar mayores niveles de eficiencia al producir mayor cantidad por unidad de tiempo y optimizar recursos al compartir espacios, maquinarias y servicios públicos. Todo esto permite a la empresa de outsourcing obtener un costo unitario inferior. Otra razón por la cual el proveedor puede ofrecer un menor precio es porque la producción se desarrolla en países que impliquen una producción de bajo costo. Inicialmente, se concebía esta posibilidad en busca de mano de obra más barata, pero ahora el interés está centrado en el servicio y en el uso de las tecnologías.

Por ejemplo, en el sector salud es común la práctica de telemedicine clinic, en la que un especialista ubicado en un país puede analizar radiografías realizadas en hospitales de otro país. Asimismo, grandes empresas como General Electric, Iмв у Cisco, trasladaron sus servicios de TI a la India. Otro ejemplo interesante son los casos de dos grandes bancos españoles: en primer lugar, el BBVA, el cual tenía su sede del back office en Madrid y traslada aproximadamente el $70 \%$ de las actividades a centros subcontratados en otras partes del mundo con menores costes, como, por ejemplo, Buenos Aires, Ciudad de México y Lima; en segundo lugar, el Grupo Santander, que en la última década ha transferido de manera progresiva el desarrollo de software, el mantenimiento de centros de cálculo y las operaciones de negocios a tres filiales en las que ha concentrado, subcontratado o trasladado parte de esas actividades (Agnese y Enric, 2011).
El outsourcing no es ajeno a las empresas colombianas. Sistecrédito es una empresa que presta servicios de outsourcing de crédito y cobranza para establecimientos de comercio como, por ejemplo, almacenes o pequeños locales. En esta medida, la empresa que contrata a Sistecrédito le vende facturas con un descuento y un plazo de tiempos pactados previamente. Entre las actividades que realiza, se encuentra el estudio de crédito, la asignación de cupos y la gestión de cartera. El servicio que ofrece Sistecrédito genera muchos beneficios para la empresa contratante, tales como no tener que realizar cuantiosas inversiones en personal, equipos y tecnología para un departamento dedicado al seguimiento y el cobro de la cartera de los clientes, así como el ahorro en los procesos legales para los clientes morosos, además de disponer del dinero mucho antes de que el cliente cancele la deuda. De acuerdo con Juan Rafael Sierra, gerente de tecnología de Sistecrédito, para los almacenes el outsourcing de crédito y cobranza es una estrategia adecuada en términos de costos y riesgos; el comerciante tiene protegida su venta, la empresa de outsourcing responde por la cartera, mejora el flujo de caja del cliente y la empresa contratante no tiene que preocuparse por los clientes morosos, ni mucho menos por los procesos jurídicos. Desde el punto de vista de mercadeo, el cliente debe volver al almacén con la posibilidad de recompra, y esto es mucho más beneficioso para el establecimiento (J.C. Sierra, comunicación personal, 13 de julio de 2016).

\section{Reducir o eliminar costos fijos}

El segundo beneficio del outsourcing es la reducción o eliminación de los costos fijos, entendidos como aquellos recursos que permanecen, sin importar si cambia el nivel de producción. Cuando una empresa deja de producir algún componente o contrata un servicio por outsourcing, elimina una gran cantidad de costos fijos. Es muy posible que los costos que quedan sean los relacionados con el precio cobrado por el proveedor, el cual solo se paga cuando la empresa requiera el componente o el servicio; si la empresa, en caso dado, no necesita el componente o el servicio, el cobro será cero. Estos costos se denominan "costos variables", es decir, aquellos que cambian o fluctúan según el volumen de actividad.

Un ejemplo de lo anterior se encuentra en una empresa de transporte, propietaria de una flota de vehículos. En esta empresa, los costos fijos están 
relacionados con aquellos que no dependen del uso del vehículo. Sin embargo, aun si estos vehículos están inmóviles, igualmente consumen dichos recursos. A este grupo pertenecen los impuestos, el SOAT, los seguros, el sueldo del conductor, la depreciación del vehículo y el mantenimiento preventivo, entre otros. Si se contrata el servicio de transporte con un tercero, entonces se tendrá un costo variable representado en el precio que cobra el proveedor y no se tendrá que pagar todo lo mencionado.

Al cambiar la estructura de costos de una compañía de fijos y variables a solo variables, se mitiga el riesgo en momentos de baja producción. Por ejemplo, una empresa que ha producido todo lo planeado pero no logra las ventas esperadas, no generará los ingresos esperados, pero sí tendrá costos, dado que son fijos e independientes de las ventas. A partir de esto, es posible que la empresa arroje un resultado muy inferior al esperado, e incluso pérdidas. Por el contrario, si la compañía tiene una estructura de costos variables y no genera ventas, entonces no se presentarán costos asociados; de esta manera, se puede dar mayor flexibilidad y la empresa podrá adaptarse al tamaño de las necesidades del mercado.

La empresa colombiana Uniformes, productora de uniformes para instituciones educativas y empresas, con más de 20 años de tradición, también se ha beneficiado de un cambio de costos fijos a costos variables. En sus inicios, el proceso de confección se desarrollaba de manera interna, por lo cual la empresa asumía todos los costos fijos; en la actualidad, el proceso se contrata con terceros. Con esta decisión se pasó de tener costos fijos durante todos los meses del año, independientemente de las ventas, a pagar un precio unitario por prenda confeccionada en los momentos en que las ventas de la empresa así lo requieran. En palabras del gerente Luis Fernando Fernández: "Para Uniformes no justifica tener planta para confección; por el tema de costos y el precio por el que se paga la prenda no justifica. Me es más rentable pagar por fuera, conseguir externos" (L. Fernández, comunicación personal, 25 de julio de 2016). El mercado de los uniformes, en especial los de instituciones educativas, tiene tres momentos anuales de alta producción: enero, abril y mayo; los demás meses son de baja producción. Al tener a las operarias de confección registradas en la nómina de la empresa durante todos los meses, era obligatorio pagar sueldos y demás componentes salariales, condición que resultaba inviable en los periodos de baja producción, cuando se disminuía la productividad y se tenía capacidad ociosa. De igual forma, al decidirse por el servicio outsourcing se liberó espacio de la planta, se redujeron los costos de servicios públicos y mantenimiento, entre otros. Con esta modalidad solo se paga por prenda confeccionada, y de esta manera se ajusta el flujo de caja de la empresa con la producción.

\section{Concentrarse en las actividades clave}

El tercer beneficio del outsourcing es que permite concentrarse en las actividades clave del negocio. Una vez las compañías definen sus actividades estratégicas, así como su tiempo, sus esfuerzos y recursos, solo se debe invertir en estas y así innovar, crecer y mantener una ventaja competitiva. El outsourcing libera a las compañías de preocupaciones innecesarias debido a que los servicios o productos que no hacen parte de lo estratégico pueden contratarse externamente. Este es el caso de Dell, compañía multinacional estadounidense, la cual se concentra en el diseño y la personalización de los equipos, pero compra todos los componentes a proveedores asiáticos, quienes los ensamblan y envían a través una empresa de mensajería.

Otro ejemplo para destacar es la Institución Universitaria CEIPA, una empresa colombiana fundada hace más de 40 años y dedicada a la educación presencial y virtual en áreas administrativas. Actualmente, esta escuela de negocios contrata el proceso de mercadeo de sus programas con la empresa EduHub, experta en la comercialización de servicios educativos. Esto fue necesario debido a que el mercadeo de alto nivel que requería era de alto riesgo y costo. El rector de esta escuela de negocios, Diego Mauricio Mazo, reconoce dos grandes ventajas en la estrategia: el tercero realiza todas las operaciones logísticas, y solo se paga por alumno matriculado (D. Mazo, comunicación personal, 14 de julio de 2016).

La ejecución de las estrategias de mercado implica muchos detalles, por ejemplo, en la realización de los eventos se requiere el staff, la logística, la comunicación y la publicidad, entre otros. Si la escuela de negocios quisiera hacerlo de forma interna, tendría que disponer de gran parte de sus procesos para dar respuesta a esta necesidad, de manera que se desvía de su razón de ser y corre el riesgo de que la inversión no logre los resultados esperados. Para Eduhub el mercadeo es su core, así que ofrecer el servicio a varias empresas 
le permite generar sinergias y distribuir los costos en todos los clientes, por lo cual su servicio se vuelve rentable.

\section{Conocimiento, experiencia y tecnología del proveedor}

El cuarto beneficio del outsourcing es que la empresa se favorece del conocimiento y la experiencia del proveedor, el cual, por sus inversiones en metodología, tecnología y crecimiento personal, es altamente especializado. Cuando una empresa elige el outsourcing, también accede al know how del proveedor, algo que, en el caso en que lo quisiera obtener de forma interna, le implicaría recursos y tiempo.

A continuación, se analizarán las empresas call center o centros de llamadas y los servicios logísticos. Las empresas call center cuentan con personal altamente cualificado para brindar una excelente atención al cliente, con la mejor experiencia de servicio y con un trato profesional y personalizado, todo lo cual impacta de forma positiva la imagen de la empresa contratante. Además, los call centers cuentan con tecnología especializada para la rápida atención de las llamadas, lo cual disminuye los tiempos de espera, evita las congestiones en la línea, las llamadas pérdidas y ofrece así un servicio extendido, incluso las 24 horas. Dadas estas condiciones, se tiene mayor control y seguimiento a las actividades, se cuenta con reportes en línea de la situación de las llamadas y las tarifas son más económicas.

DHL es una de las principales empresas de coordinación y transporte de envíos en todo el mundo. En el tema de transporte, las compañías son exigentes con el proveedor y demandan de este relaciones a nivel local, nacional e internacional; además, transportar la mercancía desde y hasta cualquier puerto marítimo o aeropuerto, $\mathrm{y}$ son muy estrictas con la entrega y la calidad en el transporte. De igual forma es importante que las empresas de transporte de envíos cuenten con monitoreo constante de sus productos, saber dónde están, qué ruta van a tomar y tener pólizas de seguro con una cobertura total de las mercancías. Debido a estas exigencias, las compañías necesitan contar con la experiencia y el respaldo suficientes de una empresa de tercerización especializada y con trayectoria. Al respecto, el gerente general de DHL Global Forwarding Colombia, Kurt Schosinsky, indica:
Hoy, los clientes solicitan soluciones a la medida, que soporten sus planes de crecimiento y expansión. Piden a su socio logístico un apoyo integral en comercio exterior, aduanas, logística nacional e internacional, transporte aéreo y marítimo, almacenamiento y distribución, así como administración de su cadena de abastecimiento desde y hacia varias regiones del mundo, a fin de hacer menos complejas las operaciones, mejorar la calidad y reducir costos. (Magri, 2017)

Otro ejemplo interesante se encuentra de nuevo en la Institución Universitaria CEIPA, la cual utiliza como learning management system (LMS) un software libre llamado Sakai, el cual requiere una estructura compleja para su administración. Cuando la Institución Universitaria CEIPA decidió utilizar Sakai, emprendió el proceso con personal interno, el cual debió instalarlo, traducirlo al español y realizar las pruebas y ajustes que se precisaban para que funcionara de acuerdo con las necesidades y los requerimientos. Durante cinco años, la Institución contó con un equipo de personas dedicado de forma exclusiva a dar soporte a esta plataforma. Pasado este tiempo, las directivas conocieron un proveedor que ofrecía servicios técnicos especializados en Sakai. Inicialmente, se contrataron sus servicios cuando se tenía algún problema mayor, pero el crecimiento exponencial y rápido de la Institución hizo del soporte algo insuficiente; se valoraron, entonces, las diferentes opciones para la administración del software y se tomó la decisión de contratar el outsourcing.

El outsourcing para el soporte de Sakai tiene una propuesta de valor en la que se garantiza que no tendrá problemas de funcionamiento y, además, que manejará un nivel de confiabilidad del 99,39\%. La Institución Universitaria CEIPA no se preocupa por tener bases de datos, por actualizar el software, contar con ingenieros especializados, data center, hardware o comunicaciones: la Institución solo requiere de un administrador que cree usuarios, grupos y genere reportes; de todo lo demás se encarga el tercero. Lo anterior permite que CEIPA concentre todo el esfuerzo en el uso de Sakai, y no en el soporte ni en el desarrollo. Además, la Institución se beneficia de la economía de escala que logra el proveedor, y puede también apropiarse de los avances y las mejores prácticas que este desarrolla. Uno de los temas más complejos en este caso fue dimensionar el nivel del servicio, en especial en los momentos de alto tráfico en la plataforma, ante lo cual el proveedor 
buscó la solución con la comunidad de Sakai para la estabilización del proceso. Este es un claro ejemplo de que el conocimiento, la experiencia y la tecnología del tercero proporcionan una solución más completa de las necesidades.

\section{Riesgos}

El outsourcing también implica una exposición al riesgo que deben revisar y analizar las empresas a fin de minimizar los impactos. A continuación, se ilustran los principales.

\section{Convertir a un proveedor en competidor}

El primer riesgo del outsourcing es que el proveedor se convierta en competidor. En este sentido, se debe reconocer que la empresa de outsourcing tiene alta experiencia en desarrollar productos para varios clientes, lo que le da una destreza en tiempos de producción y optimización de costos; además, el tercero tiene acceso a la propiedad intelectual de la empresa contratante y podría beneficiarse de esta información aplicándola en otras. Si el tercero es muy ambicioso, podría reclamar para sí los beneficios de los componentes que fabricó. El proveedor puede combinar el aprendizaje, la experiencia y la habilidad adquirida en la prestación del servicio para crear su propia empresa o línea de negocio, y cambiar su rol de proveedor a competidor.

En el sector de computadores personales, se encuentran casos de empresas que pasaron de prestar solo el servicio de ensamblaje de los computadores bajo la modalidad de outsourcing, a diseñar y desarrollar el producto, e incluso se conocen casos en los que compraron las fábricas de sus contratantes. Uno de estos ejemplos es IBM, empresa multinacional estadounidense de tecnología y consultoría reconocida por inventar el modelo líder de computadores personales. Sin embargo, no pasó mucho tiempo antes de que la empresa abandonara este segmento, para pasarlo en su gran mayoría a Lenovo, empresa fundada en 1984 como distribuidora en China de los equipos fabricados por IBM. En igual situación se encuentra la empresa Sanmina-(SCI), fabricante de algunos computadores para IBM en Estados Unidos que, finalmente, compró las fábricas en las que se producen los computadores (Aruñada y Vázquez, 2006).

Algunas recomendaciones para mitigar el efecto de este riesgo son "discreción al momento de revelar los secretos propios, cautela al momento de elegir con quiénes relacionarse, el grado cabal de intimidad, lealtad y generosidad hacia los propios socios y clientes" (Aruñada y Vázquez, 2006, p. 4).

\section{Pérdida de conocimiento y habilidades}

El segundo riesgo es la pérdida de conocimiento y habilidades, debido a que al transferir la ejecución de una actividad a un tercero se puede perder el sentido del producto o servicio a largo plazo. Si el proveedor realiza actividades de innovación, este conocimiento queda en poder de la organización y no es transferido al cliente, por lo cual la empresa pierde el acceso inmediato a la tecnología, a los productos y los procesos de última generación, descuida las mejoras y tiene dificultades para ejercer control, seguimiento $y$ apropiación de los nuevos conocimientos.

En este caso en particular, toma importancia la gestión de la información y el conocimiento, si se tiene en cuenta que el proveedor o tercero puede ser una persona natural o jurídica que desarrolla dominio sobre el cliente, a partir de la innovación y el conocimiento especializado, toda vez que el proveedor llega a tener un nivel tal de especialización que le permite asumir una posición dominante en una estrategia futura de negociación.

Una organización dedicada al desarrollo de software cuya producción, en gran parte, se dé por outsourcing, y que no genera mecanismos de transferencia de conocimiento en el tiempo, puede verse abocada a tener dificultades con la continuidad de los proyectos; esta situación se agudiza cuando se dan salidas del personal o rupturas del contrato de outsourcing.

\section{Dependencia del proveedor}

El tercer riesgo es la dependencia del proveedor. Esto implica un grado de vinculación con el tercero que limita el desarrollo normal de las operaciones; en este sentido, la empresa se puede ver afectada en temas de calidad, oportunidad, imagen y costo.

Este es el caso de la ya citada empresa Uniformes. En especial en temporadas de alta 
demanda como, por ejemplo, la de fin de año, se presentan dificultades para la contratación de la confección con un único proveedor, por lo cual la compañía contrata con varios proveedores. Como resultado de delegar la producción en varios puntos, la calidad es diferente, la logística de entrega y de recibo de la mercancía es compleja, el costo es mayor y, en algunas ocasiones, el pago es a plazos muy cortos, lo cual afecta el flujo de caja. Muchos de estos talleres los conforman quienes alguna vez fueron empleados de grandes fabricantes $y$, luego de retirarse, empezaron su propio negocio con solo tres máquinas: plana, recubridora y fileteadora. En la mayoría de los casos, estas personas no tienen la cualificación ideal, de allí que se afecte la calidad. Por otra parte, al contar con talleres pequeños, no tienen personal suficiente para atender todas las operaciones, lo que implica nuevas contrataciones que afectan el tiempo de respuesta del producto. De acuerdo con el gerente de Uniformes, durante el 2015 se registraron pérdidas de aproximadamente 200 millones de pesos, debido a que no se alcanzó a cumplir en el tiempo definido para los clientes. Esta mercancía queda en consignación hasta que nuevamente se abra la temporada, pero existen casos en los que se puede perder toda la inversión realizada.

\section{Incremento de los costos de puesta en marcha}

El cuarto riesgo del outsourcing es el incremento de los costos de puesta en marcha, es decir, todos los recursos destinados a la búsqueda, la selección, la contratación y la implementación de la tercerización.

En los costos de selección se deben considerar los recursos destinados a la evaluación de las diferentes alternativas que se tienen para la selección del proveedor; es indispensable examinar experiencia, infraestructura, cultura de calidad y oportunidad en la entrega. Los costos de negociación y contratación implican reuniones con el proveedor seleccionado para llegar a acuerdos de servicio, definición del contrato y manejo de pólizas. Los costos de implementación incluyen los tiempos de los empleados internos en la transferencia al proveedor del estilo, las normas y la cultura de la organización, así como la adaptación del nuevo proceso. Es necesario incluir en este ítem las adecuaciones en infraestructura o procesos para llevar a cabo el outsourcing y los costos de pruebas y ensayos.

A fin de ilustrar el costo de la puesta en marcha, se tomará como ejemplo una empresa de venta masiva de productos de belleza que concentra su operación en la importación de productos para la distribución a nivel nacional. En aras de comprender la dinámica del negocio, se describirán los cuatro agentes involucrados en el proceso: proveedor del producto, agente de carga internacional, agencia de aduanas y transporte local. El proveedor del producto es la empresa productora que, para el caso, es internacional; el agente de carga internacional es el encargado de la recogida y movilización a nivel internacional del producto; la agencia de aduanas es la responsable de la nacionalización y los cumplimientos legales de la mercancía; y el transporte local interviene para la ubicación de los productos en las bodegas de las empresas.

La empresa de venta masiva contaba con contratación para los tres últimos agentes (agente de carga internacional, agencia de aduanas y transporte local). Incluso, para algunos existían varias empresas proveedoras, lo que generaba grandes dificultades en el seguimiento y la trazabilidad de las diferentes actividades operativas de los procesos, con el inconveniente de que la empresa cada vez crecía más y, con ello, el número de labores. Dado lo anterior, se tomó la decisión de centralizar todas las actividades en una sola empresa que prestara los servicios integrados de logística, bajo una modalidad inhouse, es decir, en las instalaciones de la empresa. Esto conllevó a que la empresa de venta masiva proporcionara las condiciones para que la empresa de outsourcing logístico se instalara en la empresa, para lo cual se realizaron grandes inversiones en adecuaciones locativas, compra de inmobiliario y equipos de comunicación a estos espacios, programación de las jornadas de capacitación y empalme entre las dos empresas, acuerdos de manejo de los sistemas de información, así como definición de procedimientos para delimitar el alcance de una y otra empresa. De igual manera, la empresa contratante era la responsable del costo de transporte y alimentación de los empleados del outsourcing, sin contar el tiempo que la empresa se demoró en estructurar el proyecto, definir los pliegos de licitaciones, realizar la convocatoria y la selección del proveedor. 


\section{Incremento de los costos de seguimiento}

El quinto riesgo hace referencia a los costos de seguimiento, coordinación y supervisión. Los costos de supervisión deben estar orientados a un tema de control desde la prevención, evaluación y reconocimiento de fallas, bien las detecte la empresa o bien el cliente. El reconocimiento de las fallas son aquellos recursos que se asocian con defectos y errores encontrados antes o después de transferir el producto al cliente. En este ejercicio toman relevancia los costos por desechos, reprocesos, desperdicios y reinspecciones. Cuando la falla la detecta el cliente, los costos se derivan en reclamaciones, reparaciones por garantía, devolución de mercancías, demandas y mala imagen de la compañía.

De igual forma, es importante considerar los costos de finalización del contrato de outsourcing, bien sea porque la empresa reintegra la actividad o porque cambia de proveedor. En este sentido, es usual que las empresas contratantes definan tiempos cortos para los contratos con el objetivo de estar en capacidad de evaluar de forma constante la calidad, el servicio y el costo del outsourcing, y así generar licitaciones para elegir siempre el mejor proveedor del mercado; además, es una manera de contrarrestar el riesgo por la suspensión anticipada del contrato y no incurrir en multas por incumplimiento.

Un ejemplo de lo anterior se da en empresas que cuentan con un número representativo de vehículos, porque se dedican al transporte de mercancía, al transporte masivo de pasajeros o al arrendamiento de vehículos, y deciden contratar talleres inhouse a fin de mejorar el servicio de sus vehículos. Esta decisión implica la contratación de personal que realice seguimiento constante a la calidad y la pertinencia de los mantenimientos preventivos y correctivos realizados a los vehículos por parte del tercero.

\section{Afectación del clima laboral}

El sexto riesgo del outsourcing es la afectación del clima laboral. Cuando una compañía toma la decisión de implementar esta estrategia, las personas relacionadas de forma directa con la actividad son susceptibles de ser despedidas por la eliminación de puestos de trabajo que se trasladan al tercero, lo que genera entonces incertidumbre, desmotivación en los empleados y disminución de la productividad, condiciones que terminan por afectar el clima organizacional. Por otra parte, es usual que las compañías realicen movimientos organizacionales cuando toman la decisión: traslados a otras áreas, redistribución de funciones y responsabilidades, e incluso transferencia de personal de la empresa al proveedor, todo lo cual afecta las condiciones laborales.

Por otra parte, cuando se contratan los servicios outsourcing se debe tener presente que esos trabajadores no son empleados de la empresa (por lo cual su sentido de lealtad y pertenencia no será el mismo), así como las diferencias en las condiciones y las garantías del personal interno. Esta realidad genera comparaciones entre los empleados, circunstancia que también termina por afectar el clima laboral. Esta falta de vínculo del trabajador outsourcing propicia un menor compromiso e identidad. Además, no se garantiza la continuidad en la empresa y la implementación de instrumentos de recompensas o de sanciones no son procedentes.

El Hospital General de Medellín es una empresa social del Estado que presta servicios de salud en Medellín, Colombia, y la cual tiene al conjunto de auxiliares de enfermería contratados de forma directa. Sin embargo, este tipo de contratación no era caracteristica predominante en el pasado, pues antes contaba con el outsourcing de auxiliares de enfermería ofrecido por un tercero. Esta estrategia permitía alivianar la parte financiera, aunque para el área de gestión humana resultaba bastante negativa por temas de calidad y acreditación, ya que el personal outsourcing no se apropiaba de los sistemas de gestión de calidad y existían grandes dificultades para organizarlo.

Inicialmente, en un mismo proceso se mezclaban los auxiliares de enfermería contratados a través del tercero y los auxiliares de enfermería del hospital. Quienes estaban contratados por outsourcing no se sentían parte de la institución, ya que existía una brecha en temas salariales, de beneficios y de cultura organizacional. En un segundo momento se designó el personal de outsourcing de forma exclusiva para unas actividades; sin embargo, la brecha continuaba, el personal de outsourcing sentía desánimo con respecto a los beneficios extras que obtenía el personal de planta y la capacitación era muy diferente. Es así como, finalmente, la institución tomó la decisión de no 
continuar con el outsourcing y contratar a todo el personal de forma directa.

\section{Incumplimiento con las expectativas de calidad y de tiempos de entrega}

El séptimo riesgo del outsourcing es que no se cumpla con las expectativas, ya que al contratar a un tercero se corre ese riesgo en términos de precio, calidad y oportunidad. De la teoría a la práctica existe una brecha que limita el desarrollo efectivo del contrato. Si bien uno de los beneficios del outsourcing es el acceso a personal mejor cualificado, en la práctica puede suceder que el proveedor no cuente con el personal con las habilidades y las competencias para desarrollar el trabajo, y, por tanto, no cumpla con los tiempos de entrega o con la calidad del producto. Son muchas las razones para no lograr los objetivos de la tercerización y declinar; estas pueden ser de comunicación, calidad, costos, agilidad y oportunidad, entre otras.

El caso que ilustra este riesgo corresponde a una empresa dedicada al diseño, la construcción y el mantenimiento de proyectos de ingeniería eléctrica y telecomunicaciones ubicada en Barranquilla, donde los servicios de contabilidad se encontraban a cargo de un tercero que, entre sus funciones, tenía la de la elaboración y presentación de los estados financieros y declaraciones tributarias. Infortunadamente, por falta de cumplimiento en la entrega de estas últimas, la empresa debió pagar sanciones e intereses ante la DIAN por no presentar las declaraciones de dos años. En este escenario, la empresa decidió dar por terminado el contrato e iniciar la conformación del área contable dentro de la empresa, en busca de brindar seguridad y solidez en el tema y evitar los extracostos.

El incumplimiento de las expectativas puede acarrear impactos altamente negativos para las empresas en términos financieros y de reputación. Este es el caso de Toyota Motor Corporation, la compañía fabricante de automóviles más grande del mundo y respetada por su calidad. En el 2015 presentó problemas con airbags defectuosas fabricadas por la empresa Takata Corporation. Los airbags de Takata Corporation tenían la probabilidad de explotar con demasiada fuerza, expandiendo piezas que actúan como metralla. Este evento afectó 22 modelos que comercializaba la empresa en Japón, incluyendo el Corolla y el Vitz. Al menos ocho personas murieron en todo el mundo, y centenares resultaron heridas por este motivo. El caso de Toyota Motor Corporation no es el único que vincula los airbags. También se presentaron problemas similares con Mazda Motor Corporation y Honda Motor Co., Ltd. La compañía Takata Corporation fue multada en el 2015 al encontrarse que los defectos en las bolsas de aire provocaron muertes y centenares de heridos, e incluso en accidentes leves las bolsas presentaban problemas (Univisionnoticias, 2016).

\section{Conclusiones}

El término outsourcing se aplica en la administración actual como una estrategia para alcanzar una mayor competitividad al ofrecer un producto o servicio con valor agregado diferenciador que cumpla con las expectativas del cliente y los requisitos de la empresa. Si bien uno de los elementos fundamentales por lo que se recurre al outsourcing es la disminución de los costos - y en esencia fue uno de los motivadores que dio origen a esta forma de contratación-, no debe ser la única razón. A nivel estratégico, el outsourcing permite la concentración en las actividades clave, al enfocar el tiempo, los esfuerzo y los recursos en los elementos de valor, y dejar a terceros los detalles operativos. Asimismo, gracias al outsourcing la empresa se beneficia de los conocimientos y la tecnología de última generación que tienen los proveedores, lo que eventualmente favorecerá una mejor adaptación a los cambios. En el tema de la eficiencia, se busca la administración adecuada de los recursos con su racionalización a partir de la administración de los costos y la optimización de los procesos.

Las empresas cada vez están más expuestas a escenarios exigentes y competitivos que demandan de los gerentes la toma de decisiones basadas en análisis de escenarios. El outsourcing es una estrategia que se debe analizar con detenimiento, puesto que, si bien son muchos los beneficios, también genera riesgos que deben ser identificados, medidos, valorados y tratados. Por esta razón, la decisión no debe solo referenciar elementos financieros y técnicos, sino incluir también el análisis de la importancia del proceso para la empresa, la experiencia del proveedor y los acuerdos de negociación. Sí la decisión se toma sin análisis previos, puede traer como resultado altos costos, suspensión del servicio e incluso mala imagen para la empresa. 
Además, es fundamental tener claridad del impacto del outsourcing en la estrategia del negocio. Es por ello que muchos de los ejemplos aportados en la nota técnica dan cuenta de la entrega a un tercero de actividades y componentes que no son del core del negocio, para así conservar la esencia de la empresa y permitir ser diferenciada en un mercado cada vez más competitivo, al igual que minimizar la materialización de los riesgos inherentes a la decisión.

Durante la construcción de esta nota técnica se evidenció que la aplicación del outsourcing no es exclusiva para un tipo de empresa o sector: desde las empresas pequeñas hasta las grandes multinacionales implementan esta estrategia, y es posible encontrar ejemplos de casos en el sector de producción, servicios, tecnología y salud. Ahora existen mayores riesgos en empresas en las que el conocimiento es prioridad; tal es el caso de las empresas de tecnología, las cuales, al recurrir a un outsourcing, pueden perder la esencia de su negocio y terminar por formar un futuro competidor.

La aplicación del outsourcing no es estática; por el contrario, cada empresa tiene una dinámica diferente y utiliza o deja de utilizar esta estrategia de acuerdo con las necesidades de la compañía. Mientras la gran mayoría de las empresas toma como opción el outsourcing, y entrega una actividad, un componente o un servicio a un tercero, otras empresas deciden retomar el control y las dirección, pues consideran que un tercero no debe realizar las actividades de la empresa.

\section{Referencias}

Agnese, P. y Enric, J. (2011). Offshoring: ¿Y si la ventaja no solo fueran los costes? IESEinsight, 60-62.

Arruñada, B. y Vázquez, X. (2006). Cuando su fabricante contratista se convierte en su competidor. Harvard Business Review, 2-10.

Cepeda, R. I. (2012). Tercerización: una estrategia para lograr ventaja competitiva en las organizaciones. Revista La Sociedad Académica, 55-62. Recuperado de http://www.itson.mx/publicaciones/sociedad-academica/Documents/revista40.pdf\#page $=57$

Magri, A. (2017, mayo 16). En tus manos encomiendo mi logística. Revistadelogistica.com. Recuperado dehttps://revistadelogistica.com/actualidad/ en-tus-manos-encomiendo-mi-logistica/

Revista Dinero. (2014, julio 24). Un año dorado. Outsourcing. Dinero.com. Recuperado de http://www. dinero.com/especiales-comerciales/outsourcing/ articulo/perspectivas-industria-del-outsourcing-colombia/198885

Suárez, J. F. (2016, octubre 13). Movidas en BPo consolidan oferta paisa. El Colombiano.com. Recuperado de http://www.elcolombiano.com/negocios/movidas-en-empresas-bpo-consolidan-la-oferta-paisa-CB5157242

Univisionnoticias. (2016, enero 22). EE. UU. ampliaría el llamado a revisión por las bolsas de aire defectuosas de Takat. Univision.com. Recuperado de http:// www.univision.com/noticias/recalls/eeuu-ampliaria-el-llamado-a-revision-por-las-bolsas-de-aire-defectuosas-de-takata 
